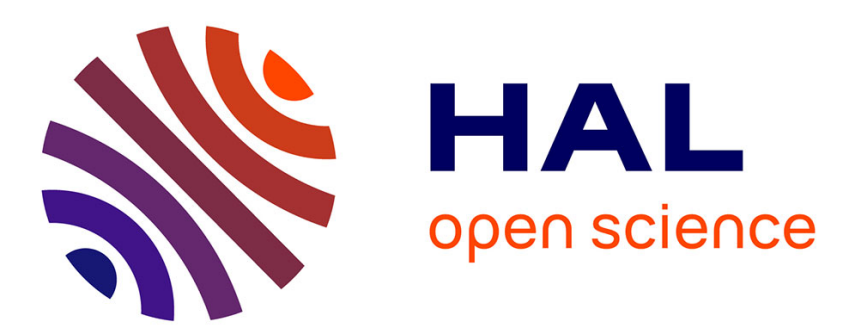

\title{
Handbook of Technical Communication
}

\author{
Alexander Mehler, Laurent Romary, Dafydd Gibbon
}

\section{To cite this version:}

Alexander Mehler, Laurent Romary, Dafydd Gibbon (Dir.). Handbook of Technical Communication. Alexander Mehler; Laurent Romary; Dafydd Gibbon. De Gruyter Mouton, pp.839, 2012, 978-3-11018834-9. hal-00786386

\section{HAL Id: hal-00786386 \\ https://inria.hal.science/hal-00786386}

Submitted on 12 Nov 2016

HAL is a multi-disciplinary open access archive for the deposit and dissemination of scientific research documents, whether they are published or not. The documents may come from teaching and research institutions in France or abroad, or from public or private research centers.
L'archive ouverte pluridisciplinaire HAL, est destinée au dépôt et à la diffusion de documents scientifiques de niveau recherche, publiés ou non, émanant des établissements d'enseignement et de recherche français ou étrangers, des laboratoires publics ou privés.

\section{(c)(1)}

Distributed under a Creative Commons Attribution| 4.0 International License 


\section{Contents}

1 Introduction: Framing Technical Communication 1

1 On the technical side of communication . . . . . . . 1

2 Overview of the book . . . . . . . . . . . . 12

2.1 Part I: basic communication technologies \& infrastructures 15

2.2 Part II: technical communication management . . . . . . . 19

2.3 Part III: communication by means of technology . . . . . 22

$\begin{array}{ll}\text { Notes } & 27\end{array}$ 



\section{Chapter 1 Introduction: Framing Technical Communication}

By Alexander Mehler, Laurent Romary and Dafydd Gibbon.

\section{On the technical side of communication}

The Handbook of Technical Communication brings together a selection of topics that range from technical media in human communication to the linguistic, multimodal enhancement of present-day technologies. It covers computer-mediated text, voice and multimedia communication. In doing so, the handbook takes professional and private communication into account. Special emphasis is put on multimodal communication, its semiotic underpinning, standardized representation, automatic processing and evaluation in the process of system development. Throughout the handbook, this is primarily done from a technological point of view that puts emphasis on the underlying processing technologies as well as on standards and formats of data representation in technical communication. Thus, basic communication technologies and infrastructures play a major role in this handbook. In summary, the handbook deals with theoretical issues of technical communication, technology-enhanced communication and its practical impact on the development and usage of text and speech as well as of multimodal technologies.

In order to give a blueprint of how the various fields of research on technical communication collected by this handbook hang together, we start from a notion of technical communication that integrates the notions of mediality, codality, modality, temporality and social complexity (see Figure 1). More specifically, we refer to five constituents in our notion of technical communication as defined below:

1. Mediality in the range of mono- and multimediality: with the rise of hypertext and hypermedia (Bush, 1945; Nelson, 1995; Engelbart, 1995; Berners-Lee, Hendler, and Lassila, 2001), multimedia 


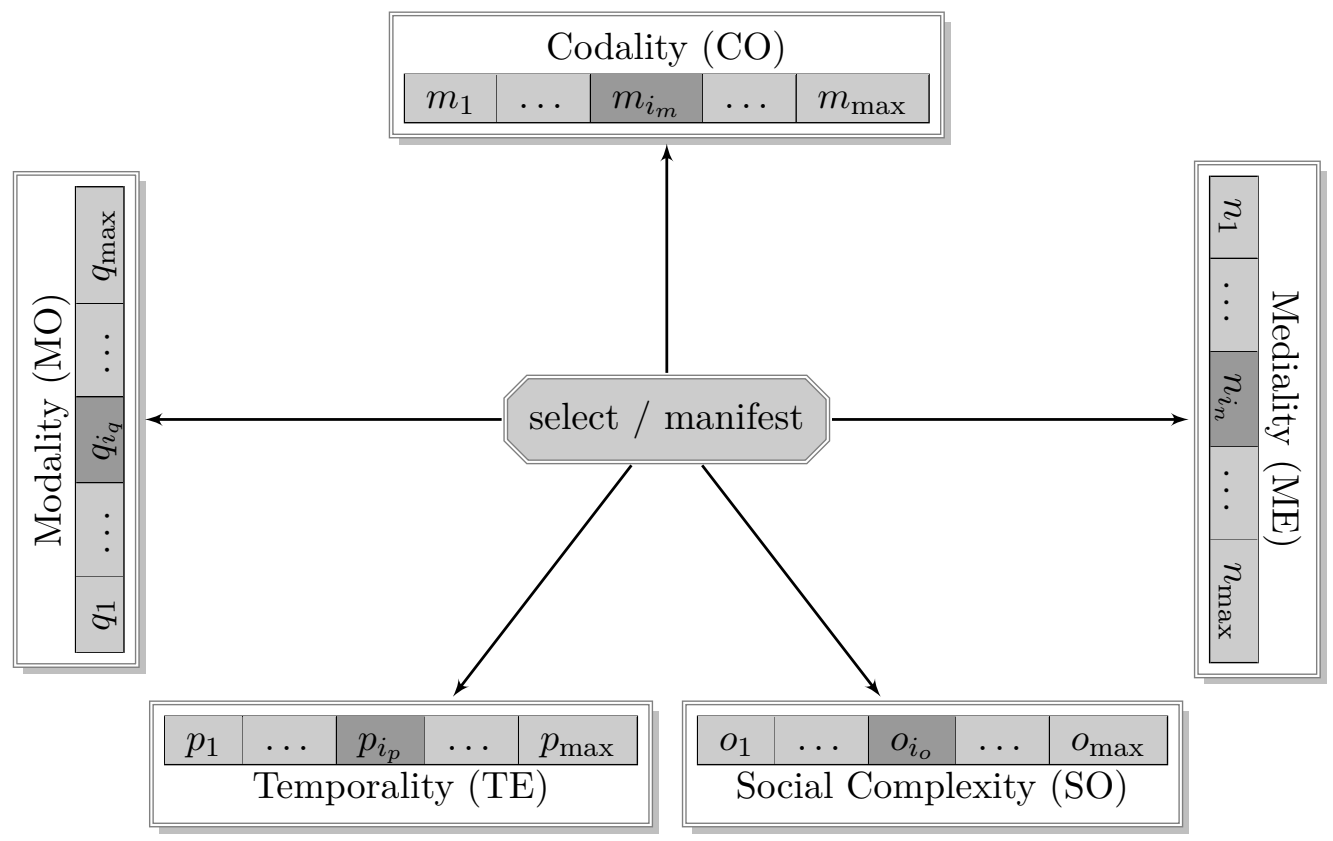

Figure 1. A five dimensional decision matrix together with a candidate manifestation in the form of the vector $\angle C O=$ $\left.m_{i_{m}}, M E=n_{i_{n}}, S O=o_{i_{o}}, T E=p_{i_{p}}, M O=q_{i_{q}}\right\rangle$. As an example consider wiki-based technical communication that is characterized by the signature $m_{i_{m}}=$ multicodal, $n_{i_{n}}=$ multimedial, $o_{i_{o}}=n: m, \quad p_{i_{p}}=$ \{quasi-synchronous, asynchronous $\}, n_{i_{n}}=$ \{monomodal production, multimodal reception\}.

has been a longstanding topic in computer science and related disciplines (only recently being complemented or even replaced by the topic of multimodality (Gibbon, Mertins, and Moore, 2000)). Generally speaking, the term medium is used in two respects: as a substance of information transmission and as a device of information storage (cf. Hess-Lüttich and Schmauks, 2004). ${ }^{1}$ However, what is stored is designated to be received in the future and, thus, storage media can be seen to serve as transmission media where the border between both readings parallels the distinction between ephemeral and persistent (synchronous, quasi-synchronous or asynchronous) transmission. An asynchronous, non-face-toface transmission necessarily requires the use of a technical de- 
vice that renders the respective communication technical. In any event, storage media do not necessarily determine the mode by which the information being transferred is processed, nor do they necessarily require a single code for encoding or decoding, respectively. Thus, we have to distinguish two more reference points.

2. Codality in the range of mono- and multicodality: traditionally, linguistics has been concerned with monocodal signs that are encoded according to a single underlying language (or code). However, not only with the advent of web-based communication and globalization we face multilingual documents that are obviously encoded by means of different codes and, thus, are multicodal. By the same token, a crossmodal supersign (Kendon, 2004) or a so called multimodal ensemble (Lücking, Mehler, and Menke, 2008) as, for example, a supersign that consists of a linguistic sign and a gesture, is multicodal (though not multilingual) where the underlying codes require at least two modes for producing and receiving its constituents. Thus, multicodal signs of this sort inevitably fall into the realm of multimodality. Another example of distinguishing mono- and multicodal signs relates to the automatic annotation of documents based on information extraction technologies (Wilks, 1997; Feldman and Sanger, 2007) that are used, for example, for automatic forecasting and algorithmic trading (Dang, Brabazon, Edelman, and O'Neill, 2009). In these cases, an algorithm based on some language model implements an additional code that, if being successful, is used to enrich the input documents informationally. It may be a question at issue whether annotated documents are multicodal or just based on multiple authors that are partially artificial. In any event, one has to consider that algorithms do not necessarily use the same code as human authors even if the output of the algorithms is input to algorithmic trading and, finally, to decision making by humans.

3. Modality in the range of mono- and multimodality: the modality of a communication concerns the sensory interfaces that the communicators use to perform this communication. In this sense, a communication is called multimodal, if at least one communicator 
uses at least two different sensory interfaces for sign production or reception. ${ }^{2}$ One may argue that any communication is multimodal since one never uses the same interface for both sign production and reception. However, if we make an additional distinction it is evident that monomodal production and monomodal reception are quite common.

Note that we call a communication (i) mono- or multimodal, (ii) mono- or multimedial, (iii) mono- or multicodal where the reference point of such attributions is (i) the sign producer or recipient, (ii) the transmission or storage medium being used and (iii) the sign system(s) being manifested (cf. Weidenmann, 1997). Note also that we may speak of multimodal, multimedial or multicodal signs: on the token level this means to select one of the latter reference points in order to stress the singularity or multiplicity of the codes, media or modes manifested by the tokens under consideration.

The three reference points of classifying communications introduced so far are, so to speak, orthogonal. This is demonstrated, for example, by multimedial documents that consist of graphically manifested texts and sound tracks that can be received in a monomodal fashion if the texts are read aloud by means of some speech synthesis. Further, a multimodal discourse in which gesture accompanies speech can be digitized and transferred by means of a single medium that captures both the visual and acoustical data stream. Thirdly, a multilingual instruction manual can be monomedially stored and monomodally received. It is evident how we may extend this list of examples that keep the communicators, transmission media and codes apart as different reference points of classifying communications. We continue this classification by means of two additional reference points:

4. Temporality in the range of synchronicity, quasi-synchronicity, and asynchronicity: none of the reference points distinguished so far explicitly refers to the notion of time as being constitutive for any act of communication. However, in reviewing the classical model of conceptual speech and writing as introduced by Koch and Oesterreicher (cf. Koch and Oesterreicher, 2001), Dürscheid (2003) stresses the need to distinguish what she calls 
mediality, that is, what we call temporality. The reason for this notional add-on is to distinguish communication situations along the synchronicity or asynchronicity of turn-taking. Along this line of reasoning, Dürscheid distinguishes between synchronous communications (of immediate or even overlapping turn-taking in shared communication situations), quasi-synchronous communications (in which turn-taking is marginally stretched so that feedback among communicators is slightly restricted), and asynchronous communications (where the communication situation is significantly stretched so that a common frame of communication can hardly be assumed). Below we shall see how this criterion of temporality combines with those of mediality, modality and codality. However, before we do that, we need to make a final distinction.

5. Social complexity in the range of monades, dyads, triads, and large social groups (aka communities): Traditionally, linguistics had to do with written documents that were mainly produced for mass communication (Wehner, 1997) where single text producers addressed large social groups of communicators (Brinker, Antos, Heinemann, and Sager, 2000). During the 1970's, dialogical communication came into the fore of linguistic research, consequently focusing on dyads of interlocutors (Brinker and Sager, 2006). Even more recently, linguistics started looking at triads or tiny groups of persons that together perform so called multilogues (Ginzburg and Fernandez, 2005). Thus, we observe a rise in social complexity that starts from monologues and dialogues to finally reach the level of so called multilogues. What is missed in these approaches is a more comprehensive account of the social complexity of communication in terms of one-toone, one-to-many and many-to-many relations as they are prototypically observed in face-to-face communication $(1: 1)$, mass communication $(1: n)$, and web-based collaboration $(n: m)$. Any account of technical communication has to consider this social dimension (cf. Zerubavel, 2003) - otherwise it fails to clarify the peculiarities of technological enhancements of, say, collaborative writing as currently provided by wiki-based systems (Leuf and Cunningham, 2001; Stegbauer, 2009). With the rise of web-based 
communication, we observe an increase of the social complexity of communication that has to be reflected by any account of technical communication.

One may ask why such a high-dimensional, parameter-rich space is needed to clarify the notion of technical communication. The reason is that technological means of communication have pervaded human communication, modifying it in various, but insufficiently classified, ways. By addressing one of these five reference points we can classify different approaches to technical communication by their semiotic differentia specifica. In order to see this, we consider three combinations of these reference points that are accompanied by specific technologies that render the corresponding communication technical in a very specific sense:

1. The case of collaborative writing: wikis (e.g., Wikipedia) allow for a form of collaborative writing that uses distributed computerbased media for producing, storing, transferring and receiving the contributions of their "wikilocutors". From the point of view of reception, this sort of collaborative writing is primarily monomodal since contributions of wikilocutors are mainly textual. Exceptions occur, for example, in the form of sound tracks or when speech synthesis is used for reading written contributions aloud so that in principle we face a situation of multimodal reception. Further, in large Wikipedias (e.g., the English or German ones) that contain citations in many languages, multicodality is the norm. In terms of temporality, wikis allow for quasi-synchronous as well as asynchronous communication: in the former case an author's contribution may be removed or further processed quickly by her collaborators, while in the latter case a textual contribution may stay unchanged for years before being modified by other wikilocutors. This temporal peculiarity of wikis relates to their dual nature as storage and transmission media (as, e.g., in the case of highly disputed discussions (Wattenberg, Viégas, and Hollenbach, 2007; Laniado, Tasso, Volkovich, and Kaltenbrunner, 2011; Gómez, Kappen, and Kaltenbrunner, 2011)). However, what makes wikis an exceptional case of technical communication is their social complexity, which ranges from manifesting mass 
communication (where articles are authored by single authors, but received by large audiences (Mehler and Sutter, 2008)) to collaborative writing and edit wars (where articles are authored by many authors in the role of content providers, removers or defenders (Brandes, Kenis, Lerner, and Raaij, 2009)). Here, technology plays the role of enabling a sort of collaborative writing where, in principle, communicators can work on the same texts at different points in time at different locations - irrespective of how distant they are in terms of space and time. That is, wiki technologies are not just a means for externalizing our social memory of texts and their intertextual relations (Sager, 1997). They also allow for suspending the time-space continuity of faceto-face communication (Zerubavel, 2003) in terms of multilogues. They do so by enabling a multitude of communicators to adopt both the role of text recipients and text producers concerning the same texts irrespective of their time-space discontinuity. Thus, we may speak of an externalization of collaborative sign processing in social groups that is enabled by wiki technologies and that extends immediate peer-to-peer learning (Rieger, 2003) to mediate collaborative learning in groups. This example shows that beyond the trias of codality, mediality and modality, temporality and social complexity are indispensable reference points of characterizing the peculiarity of technical communication as enabled by wikis. Of course, collaborative story-telling and social memorizing existed before the advent of wikis; however, wikis allow for externalizing these processes in terms of quasi-synchronous and asynchronous collaborative writing. Obviously, this is a step forward in the development of technical communication.

2. The case of ubiquitous computing: The term 'ubiquitous computing' means, literally, 'computing anywhere', sometimes also 'computing everywhere' or 'omnipresent computing' (see Chapter 21, 'Ubiquitous Computing' for a comprehensive overview of this topic). The field of ubiquitous computing arose with the emergence of small mobile computing devices such as laptop computers, PDAs (Personal Digital Assistants) and multifunction cellphones in the 1990s. In the meantime the field has developed into a very broad spectrum of interactive wireless systems 
with internet-connected smartphones, cameras and audio players, GPS-based location coordinating software applications, automatic RFID (Radio Frequency Identification) shopping checkout, and industrial, military and domestic 'smart environment' control by means of proximity detection of moving agents. Each of these different kinds of ubiquitous computing uses a different specification of the 5-dimensional decision space of classifying technical communication as presented in Figure 1. For example, collaborative game players using smartphones will typically work with the following (simplified) specification: they preferably communicate synchronously (temporal complexity) (of course, smartphones also allow for quasi-synchronous and even asynchronous communication) using touch-activated screens (multimediality) in order to produce and process signs in different (audio, visual and tactile) modes (multimodality) in a competitive, at least dialogical $(1: 1)$ or even multilogical $(n: m)$ manner (social complexity). What is characteristic for this example of ubiquitous computing is the enhancement that it provides for multimodal computing especially in situations of quasi-synchronous communication. In these situations, the space continuity of the interlocutors is suspended. Ubiquitous computing implies more than just particular technologies, however. In the technical coding dimension, ubiquitous computing is dependent on standardization of interoperable interfaces and inter-device as well as humanmachine communication protocols (see the discussion of aircraft and train control in Chapter 12, 'Verbal Communication Protocols in Safety-Critical System Operations'). Further, in the social dimension, the 'invisible' or 'implicit' identification procedures which are often involved in ubiquitous communication raise problematic ethical issues of technology-driven changes in privacy concepts, which are the topic of continuing controversy. In any event, ubiquitous computing stresses the multimodal variety of communication thereby opening up many new areas of application of technical communication.

3. The case of adaptable hypermedia: The third example covers so called adaptable hypermedia (Brusilovsky, 1996). ${ }^{3}$ These have been proposed as a class of dynamic hypertexts that allow for 
their continual adaptation subject to their usage. An example of such a system has been given by O'Donnell, Mellish, Oberlander, and Knott (2001) who argue for hypermedia systems that adapt even the wording of single hypertext modules (Storrer, 2002) to the needs, usage habits or interests of their recipients (so that, for example, beginners are provided text that is accessible at a high level of readability). As a result, different hypertexts are generated for different readers that may reflect the social, cognitive or situational specifics of their usage - with respect to form and content. Even though this is a historical example (since this technology has been replaced by a completely different development of the web), it is worth considering the kind of complexity of this sort of technical communication according to Figure 1. Evidently, adaptable hypermedia are multimedial and require multimodal reception by definition. However, they are simple as far as their social complexity is concerned: although single users can influence the generation of hypertexts being presented to them, this mainly works in terms of a filter mechanism for which the system specifies the space of possible states it can take up in advance. As a consequence, an adaptable system of this sort hardly enhances communication among different users. If at all, this relates to modeling types of users (e.g., beginners, experts etc.) and, thus, to a sort of typological interrelation of users who are supposed to "interact" in similar ways with the system (Mehler, 2009). From this perspective, it is no surprise that the web took a different development toward collaborative media as exemplified by Wikipedia. In any event, there are recent developments that aim at unifying the semantic web (Berners-Lee, Hendler, and Lassila, 2001) (see also Waltinger and Breuing (2012) in this volume) and wikis (Leuf and Cunningham, 2001) in terms of so called semantic wikis in order to allow for a kind of collaborative writing under the control of a semantic model as provided by semantic web technologies (Schaffert, Bischof, Bürger, Gruber, Hilzensauer, and Schaffert, 2006; Völkel, Krötzsch, Vrandecic, Haller, and Studer, 2006; Hammwöhner, 2007; Baer, Erbs, Zesch, and Gurevych, 2011). This may end up in a revival of notions of adaptive hypermedia under the header of the semantic web and Web 2.0 technologies. 
These three examples stress different reference points of the complexity of technical communication as shown in Figure 1: wikis point to the social complexity of collaborative writing while ubiquitous computing relates to the complexity of multimodal interfaces. Finally, adaptive hypermedia and semantic wikis emphasize the potential for development of technical writing even in settings with a focus on classical, monomedial texts. Any such development raises the question for the combinability of manifestations along the reference points distinguished in Figure 1. Equivalently, any such development challenges the limits of multimodal fusion and fission as well as of the codal, medial, temporal and social complexity of communication. The present handbook's chapters provide insights into such a drawing of boundaries.

Note that technologies are a means to resolving constraints of our embodiment (cf. Eco's notion of prostheses in Eco, 1988). Technologies are also a prerequisite for making available even the ephemeral traces that communication leaves behind - regarding any of the reference points considered so far. That is, without technical media for transferring or storing these traces, research, for example, on the social complexity of collaborative writing, on the conversational unfolding of microblogging or on the alignment (Pickering and Garrod, 2004; Rickheit, 2005) of interlocutors in multimodal face-to-face communication (Ruiter, Rossignol, Vuurpijl, Cunningham, and Levelt, 2003) would hardly be possible. Because technical communication is an indispensable channel still under development, it is now possible to study communication to its full semiotic extent - as described and foreseen in the comprehensive handbook on semiotics edited by Posner, Robering, and Seboek (1996).

In order to systematize our examples just given look again at Figure 1. It shows the reference points of classifying communication situations as considered so far. Our basic idea is to call a communication technical if any of the attribute values selected along these reference points is affected by some technology (e.g., enhanced, replaced, complemented, augmented, accelerated, controlled, managed or otherwise modified). ${ }^{4}$ That is, the technologies in use may primarily focus on enhancing the reception or the production of signs (as in the case of barrier-free communication - see Kubina and Lücking (2012) in this handbook) (i.e., the aspect of modality). Alternatively, they may focus on enhancing the storage and transmission of data (as in the case of semantic infor- 
mation retrieval - see Paaß (2012) in this handbook) (i.e., the aspect of mediality in Figure 1). Further, technologies may essentially address the problem of handling documents that contain signs of multiple languages or to adopt monocodal texts to the needs of different language communities (as in the area of multilingual computing - see Sasaki and Lommel (2012) in this volume) (i.e., the aspect of codality). Further, we have to think of digital curation (see Lee (2012) in this handbook) that seeks technologies that allow for using present-day digital artifacts (e.g., computer games, wikis, or blogs) by future generations even under comparable conditions subject to which they are currently used (i.e., the aspect of temporality). Obviously, this is an extreme example of the temporal complexity of technical communication, where the addressee of the communicative act is yet not alive. Finally, the main focus of technologies in technical communication may relate to the social complexity of communication (as in decentralized social networks and wiki media - see Diewald (2012) and Waltinger and Breuing (2012) both in this volume) (i.e., the aspect of social complexity).

Of course, technologies may provide enhancements along different dimensions at the same time and hardly realize a single such focus. Such a multiple classification is reflected in Figure 1 by example of wikis that manifest a certain selection of attribute values along our list of five reference points (see above). This results in a signature of the peculiarity of wikis in terms of technical communication. The idea is to perform the same classificatory procedure for any other sort of technical communication. As this is an open development, no one can foresee what combinations will become relevant and what further reference points will be needed to account for the rapid enhancements and modifications of our every-day technical communication. In any event, this handbook intends to provide a snapshot of this ongoing development. It collects contributions to the following areas of technical communication:

- technical foundations of technical communication

- standardization

- linguistic computing

- multilingual computing 
- multimodal computing

- talking computers (speech recognition, speech synthesis)

- language and speaker identification

- artificial interactivity

- barrier-free communication

- assistive technologies for motor and sensory communication impairments

- distributed social networks

- peer-to-peer communication

As any non-wiki-based handbook necessarily makes a selection of certain topics, we hope the reader will find our selections rich and thought-provoking, even if we have not included every relevant theme. We believe in collaborative writing and ask the reader to follow the references given at the end of each chapter and to consult Wikipedia as a continually enhanced encyclopedia that because of its temporal and social scale can better document recent developments in the rapidly changing field of technical communication. In any event, the present handbook may help to get first insights and references for some of the main fields of research in this area.

\section{Overview of the book}

The handbook on technical communication is divided into three parts and 22 chapters. While Part I focuses on technologies, the management of communication and its technology-based enhancement are dealt with in Part II and III respectively.

Part I starts with overviewing basic technologies that are used in various fields of technological communication. This includes technologies for document representation and document analysis as well as technologies for handling multimodal data and speech data in technical systems that accompany, augment or even enhance oral and other kinds 
of face-to-face communication. Part I distinguishes between the generation (Chapter 1), the representation (Chapter 2, 3 and 4), the classification (Chapter 5) as well as the management and retrieval of monoand multimodal documents (Chapter 5 and Chapter 6). It mirrors the two-fold nature of data in technical communication, which, historically, started with text-based systems in order to enter the full range of multimodal communication between humans and their artificial companions. Special emphasis is put in Part I on resources - such as corpora and machine readable dictionaries - for training, testing and evaluating technical communication systems (Chapter 7). The thorough evaluation of these systems by means of automatic, semi-automatic or purely intellectual procedures is dealt with by a special chapter (Chapter 8). Part I is the most technical part of the handbook: some of its chapters can be read as thorough introductions to the respective field of research with a focus on representation formats or on state-of-the-art technologies for processing corresponding data. All other chapters of the handbook take profit of these introductions by referring to these selected chapters as references for the respective technologies. As it stands, no handbook can give comprehensive overviews of all relevant fields of research or application. In any event, the interested reader is asked to consult the technical literature cited in these chapters to get further information.

Part II of the handbook covers seven chapters that focus on the management of technical communication in different application areas. These areas range from lexicography (including terminology and ontology management) via multilingual communication, and scientific communication to the usage of controlled languages in risk and security management. Ontologies have been invented as representation formats that enable automatic, semantic (inference-driven) data processing. Hence, ontologies play an important role in any area of technical communication that has to do with processing linguistic data (Chapter 9). In any event, processing linguistic data has to face the variety of languages due to the globalization of technical communication. Thus, Part 2 puts special emphasis on multilingual computing (Chapter 9). This includes the localization of technical systems, that is, procedures by which content and software systems are provided in multiple languages. Beyond multilinguality, multimodality is one of the areas of current development of technical communication whose semiotic underpinning is 
described in Chapter 13. This chapter complements the more technological basing point of multimodality as provided by Chapter 6 in that it looks at multimodal communication strategies in human-machine communication. Chapter 11, Chapter 12 and Chapter 14 give overviews of three areas of the management of technical communication. This tripartite overview starts with scholarly communication (Chapter 11) with a special emphasis on digital libraries and current developments in scientific communication. It is complemented by an example-driven survey of technical communication in safety-critical systems (Chapter 12). With the advent of the web, ever new technical media for communication arise. Most recently, this includes so called decentralized online social networks. The handbook reflects this very important and still upcoming area of technical communication by means of Chapter 14. Last but not least, technical media can be seen as devices not only for externalizing the human memory, but also for archiving semiotic data for future generations (see above). Chapter 15 is about this aspect of technical "communication" between the past, the present and the future. It does so from the point of view of digital curation, that is, the technical management of artifacts for future use.

Part III complements the handbook by surveying 7 areas of technologyenhanced communication. The first of these chapters focuses on Internetbased communication and Web 2.0 technologies together with their impact on technical communication and documentation (Chapter 16). Technology-enhanced learning and especially computer-assisted language learning is the topic of Chapter 17. A central aspect of technologyenhanced communication relates to the communication of human beings with artificial agents. This is the topic of Chapter 18 and 20 . Both of these chapters continue the handbook's subtopic multimodal communication, however, with an emphasis on multimodal fusion (in the course of understanding multimodal data) and multimodal fission (in the course of producing multimodal data). A classical area of technology-enhanced communication is barrier-free communication that is dealt with by Chapter 19. Starting from the comprehensive overview on multimodal communication given in this handbook, this chapter distinguishes handicaps and impairments of human beings together with corresponding technologies for bridging them in communication. Two novel developmental branches of technical communication are given with ubiquitous computing (Chapter 21) and P2P-computing 
(Chapter 22). Whereas the former accounts for the pervasion of computing technologies in everyday activities, the latter utilizes the notion of agent or peer networks for the distributed processing of data, say, in information retrieval. The handbook ends with these two application areas. They exemplify upcoming areas of human communication that once started from non-technology-enhanced face-to-face communication in order to reach the area of cloud computing, artificial interactivity and related fields of application where even machines can serve as turn-takers. Obviously, this is an ongoing development whose destination is not foreseeable.

In the following sections, the 22 chapters of this handbook are summarized in detail.

\subsection{Part I: basic communication technologies \& infrastructures}

In Chapter 1 ("Document authoring: from word processing to text generation"), Aurélien Max presents the various methods that have been progressively developed to accompany the authoring of text in technical domains. Articulating the topics around the document creation workflow, the chapter tackles all aspects of document control, from purely formatting (or structural) aspects to the linguistic checking of content. A last section addresses the issue of natural language generation whether fully automated or interactive. Together with Chapter $2,3,4$ and 5 of this handbook, this chapter is mainly about written communication with a focus on monomedial documents.

Chapter 2 by Sebastian Rahtz ("Representation of Documents in Technical Documentation") covers the various aspects of the representation of documents in digital formats. Starting with an analysis of the various levels at which a document can be construed, and identifying the correlation between the syntactic (and layout) representation of a document with its underlying semantic, Sebastian Rahtz surveys the various existing formats and standards currently deployed in the industrial and the academic communities (TeX and XML based formats such as the HTML, TEI, DocBook, OpenXML or ODF).

Complementing the preceding chapter, Chapter 3 ("Foundations of markup languages") by Maik Stührenberg provides an in-depth presentation of XML technology, as used in a variety of representational 
contexts. Starting with the notion of a semi-structured document and eliciting the class of digital objects that can be thus represented, the author shows in detail the theoretical and technical background of the XML recommendation, addressing in particular issues related to expressivity (overlapping, pointing) and parsing (schema languages).

In Chapter 4 ("Controlled language structures in technical communication"), Thorsten Trippel reflects on the various domains where controlled languages maybe used to enforce unambiguous meaning in technical communication. After an overview of the traditional domain, e.g. technical writing, where controlled languages have been in use for years, the author covers more recent aspects where digital content management requires the handling of controlled languages to ascertain the semantics of digital content. Covering issues related to controlled vocabularies, metadata languages and data categories for data modeling, the chapter provides a systematic link with the corresponding standardization activities. Although this chapter is mainly about written communication, controlled languages are also more and more important for enhancing multimodal communication by technical means, a topic that is thoroughly treated by several chapters in this handbook.

In Chapter 5 ("Document Classification, Information Retrieval, Text \& Web Mining"), Gerhard Paaß surveys state-of-theart technologies of data mining and information retrieval with a focus on textual units. The chapter deals with quantitative models of documents in written communication and their utilization for various tasks such as text classification, topic detection and tracking, topic clustering and labeling, word sense disambiguation, co-reference resolution, relation extraction and semantic retrieval. The latter technologies are called semantic as they do not require that search queries and retrieved documents share search terms; rather, they are required to contain semantically related or at least similar terms where semantic similarity is computed in terms of a distributional approach (Harris, 1954; Landauer and Dumais, 1997; Turney and Pantel, 2010). In such a way, the chapter touches a deep problem in technology-enhanced communication where the technology needs to "understand" the semiotic output of human beings in order to better serve their needs. As it stands, monomodal, monomedial written communication (including multicodal documents) will be the first area of real progress in solving this task. Consequently, 
the chapter includes the rising area of ontology mining. In so doing, it provides a reference for the interested reader for any related chapter on lexical resources, controlled languages, terminologies and lexicography within this handbook. ${ }^{5} \mathrm{~A}$ central part of the chapter relates to the distinction of supervised and unsupervised machine learning. In this regard, the chapter surveys standard methods in text and web mining. Starting from the automatic preprocessing of documents, this includes, amongst others, clustering methods, kernel methods and conditional random fields that are used, for example, for automatically annotating text constituents (e.g., as named entities) in written communication. This sort of semantic annotation is an indispensable step towards advanced technologies, for example, in technical documentation, multilingual computing or, more generally, in computational humanities. It is called semantic as the information being annotated concerns the semantic interpretation of text constituents. Advancements in technical communication will critically depend on achievements in automatizing such interpretations that heretofore constituted the domain of human expertise. ${ }^{6}$

The topic of multimodal communication, i.e. communication with more than one human output-input channel such as speech and gesture, is pervasive in the Handbook, and addressed in Chapter 6 ("Multimodal and Speech Technology") from the technological perspective by Jean-Claude Martin and Tanja Schulz, combining the issue of multimodality with that of multilinguality. Language specific problems such as typological differences in sound and writing systems and word types, and technological problems such as the lack of data and missing orthographic standards are discussed and technical strategies for solving these problems are presented. Spoken dialogue systems are addressed in detail, as well as new areas such as the recognition of emotion, personality, workload stress and multimodal speaker recognition and tracking. Finally, approaches to and applications of multimodal interfaces are discussed.

Technical communication systems require resources for development in the form of raw data, systematized data, tools for data processing and standardized archiving. In Chapter 7 ("Resources for Technical Communication Systems"), Dafydd Gibbon defines technical communication systems as devices or device networks which intervene in the communication channel between speaker/writer and addressee. 
The chapter also details resource problems from the point of view of standardization requirements, from names for languages to intellectual property rights (IPR). Typical technical communication systems are listed, as well as the kinds of data required for their development and intellectual resources such as notations and transcription systems and storage formats. Two areas are selected for detailed exemplification: resources for lexicon development and resources for developing spoken language systems and multimodal systems.

Part I is concluded by Chapter 8 ("Evaluation of Technical Communication") by Peter Menke. It provides an overview of related topics and methods with a focus on technical communication. In this way, Chapter 8 can be read as a complement to any of the handbook's chapters (in any of the areas distinguished by Figure 1) since evaluation is indispensable whenever it comes to assessing the quality of technical enhancements of communication. Starting from a notion of communication that distinguishes agents, medium and content as reference points of its technical enhancement, this chapter provides a typology of different objects of evaluation in technical communication. Further, with the help of model theory, the chapter describes evaluation as a measurement operation based on which notions (like objectivity, reliability and validity) can be explained (for more details on these topics from the point of view of linguistics see Altmann and Grotjahn, 1988; Altmann, 1993). This is the background of the third part of the chapter, where qualitative and quantitative methods of evaluation are described. On the one hand, this includes questionnaires, interviews and expert reviews, and on the other hand, statistical measures. Special emphasis is given to measures of effectiveness as used in automatic classification (cf. Chapter 5). The chapter concludes with classification scenarios showing that more often than not evaluation is rather an ongoing process than a point measurement. Since the transparency and acceptability of technically enhanced communication become more and more important not least with the advent of decentralized social networks (cf. Chapter 14), evaluation will have a strong impact on future developments in any of the areas dealt with in this handbook. 


\subsection{Part II: technical communication management}

Serge Sharoff and Tanja Schultz address in Chapter 9 ("Lexicography, terminology and ontologies") the creation and representation of lexical structures based upon a semasiological (concept to term) principle. Starting with the basic concepts issued from the semiotic domain and the early work of E. Wüster, they provide an overview of a) the characteristics of semiological structures seen as ontological systems and b) of the various technological answers to the representation of such data, in particular within ISO committee TC 37 (with TMF and TBX). In the last section of the chapter, the authors present the state of the art in terminology extraction from text and show the importance of corpora as a basis for the construction of termbanks.

The issue of multilingualism (i.e., multicodality in terms of Figure 1) in scientific communication is presented in Chapter 10 ("Multilingual Computing"), where Felix Sasaki and Arle Lommel cover the various technologies, together with the corresponding international standards that have accompanied the development of multilingual computing over the years. From character encoding (ISO 10646 and Unicode) to the various methods of representing multilingual information and segments (terminologies with ISO 16642 and TBX, translation memories with TMX, localization documents with XLIFF), the authors clarify how these methods compare with the actual processes that are required when dealing with multilingual content.

Chapter 11 by Laurent Romary ("Scholarly Communication") tackles the role of scholarly publication in the research process and looks at the consequences of new information technologies in the organization of the scholarly communication ecology. Covering issues related to document acquisition and open access models, the chapter broadens to include research data, and ends up by presenting a possible scenario for future scientific communication based on virtual research environments. In this sense, the chapter addresses both the social complexity of collaborative scientific communication and the dissemination and retrieval of multimedia scientific data.

Central issues for technical communication systems concern the issues of fitness for purpose, including reliability, risk assessment and safety in deployment environments. In Chapter 12 ("Verbal Communication Protocols in Safety-Critical System Operations"), 
Peter Ladkin treats the specific domain of verbal communication protocols in this context, with particular reference to communication involving decisions taken before airline crashes, and to train control protocols. For each of these domains case studies are provided. In the aircraft domain, communication preceding an aircraft collision (the 2002 Überlingen mid-air collision), involving conflicting decision criteria from humans and technical systems and a case where technical information was interpreted incorrectly by the crew (Cali) are discussed in detail. In the train domain, the logic of two-way train operation on single lines and the consequences of failure to follow this (Warngau) are analyzed. Solution strategies for handling these communication issues are discussed in terms of formal grammars for protocol implementation. Safety-critical situations as considered in Chapter 12 are extremely important also from the point of view of multimodal communication this topic bridges to the next chapter.

In Chapter 13 ("Multimodal Communication"), the topic of multimodal communication is addressed from a linguistic point of view by Jens Allwood and Elisabeth Ahlsén, starting with a clarification of the production, reception and function of multimodal signs in interactive contexts. Differences between human-human and human-machine communication are discussed in terms of the relative flexibility of human adaptation and the relative inflexibility of machine adaptation, cultural variation and the complex multiple channels of human communication, including gesture, posture, gaze and facial expressions. Communication control and monitoring issues such as turn management are outlined, and examples of machines involved in multimodal manmachine communication are given, ranging from computers and smartphones to robot interaction and tutoring systems. Finally, the ethical issue of apparent naturalness in machine communication systems leading to over-confidence in the abilities of the system, and responsibility for consequences of actions of the system are emphasized.

In Chapter 14 ("Decentralized Online Social Networks"), Nils Diewald deals with Social Network Sites (SNS) as one of the most recent developments of online technical communication with a great influence on social inclusion and exclusion. SNSs provide a technology to build Online Social Networks (OSN). These are social networks that are spanned among agents according to their communication relations as manifested by the underlying SNS. In this sense, we may speak of 
a social community as an OSN that is basically networked by means of the communication acts of its members as performed, for example, with the help of an SNS such as Facebook. From this point of view, Chapter 14 focuses on the social complexity of (quasi-synchronous or even asynchronous) technical communication that may include cases as simple as 1:1 communication up to the level of situations in which many members of the same community react upon the tweets or posts of many other members of the same community. However, since this sort of communication happens online, aspects of multimediality are also at the core of this chapter. The chapter is divided into three parts. It starts with discussing sociological aspects of SNSs and OSNs. This mainly includes quantitative models based on social network analysis (Wasserman and Faust, 1999; Newman, 2010). Note that this section also includes a discussion of commercial aspects of SNSs. The second section is devoted to the architecture of SNSs and its impact on issues of (de-)centralization, participation, privacy and legacy of communication via SNSs. The chapter continues with a description of technologies that underly SNSs mainly in terms of protocols and formats used to technically manifest online communication via SNSs. A last section is devoted to recent projects on SNSs that certainly will attract more and more attention with the rise of SNSs and OSNs as scientific objects of communication theory.

With Chapter 15 ("Digital curation as communication mediation"), Christopher A. Lee introduces the specific aspect of the management of digital content from the point of view of maintenance and preservation. Digital curation, seen as the selection, representation and storing of digital traces, is contemplated here from both communicative and technical activity. On the basis of the OSI (ISO 1994) model, the author covers the various levels of representation that have to be considered in digital curation, ranging from bit stream processes up to complex clusters of digital objects. Interestingly, digital curation addresses the preservation of ultimately any aspect of the complexity of technical communication as distinguished by Figure 1. Therefore, it can be seen as an outstanding research area of technical communication. ${ }^{7}$ 


\subsection{Part III: communication by means of technology}

Part III starts with Chapter 16 ("Internet-based Communication") by Ulli Waltinger and Alexa Breuing. It does so with an emphasis on semantic web standards and technologies (e.g., RDF, OWL) and the Web 2.0. This also relates to the notion of crowdsourcing (according to which efforts in data annotation and semantic tagging are delegated to web communities) that may include the integration of automatic and human computation (e.g., in the framework of games with a purpose (Ahn, 2008)). An example of crowdsourcing is collaborative writing as not only exemplified by Wikipedia (Leuf and Cunningham, 2001; Stegbauer, 2009) but also by numerous wikis that serve, for example, scientific communication, leisure communication or enterprize communication. Starting from an overview of the proliferation of technologies of web-based communication (including, amongst others, e-mail-based communication, instant messaging, and voice over IP), the chapter discusses several challenges of this field of research. This includes the notion of the web of data (cf. the linked data paradigm (Chiarcos, Nordhoff, and Hellmann, 2012)) as well as models of social and emergent semantics (Steels and Hanappe, 2006; Gabrilovich and Markovitch, 2009; Egozi, Markovitch, and Gabrilovich, 2011). In this sense, Chapter 16 can be read as an overview of the development of social complexity in web-based communication starting from mass communication (where users tend to be passive recipients of static web content), and advancing now to the area of true $n: m$-communication as exemplified by collaborative writing and microblogging (where users perform both roles as authors and recipients).

Chapter 17 ("Tutoring Systems and Computer-Assisted Language Learning (CALL)") by Henning Lobin and Dietmar Rösler deals with applying technical communication as a means to assist and enhance processes of learning. It starts with enumerating aspects under which language learning (as a special sort of communication between teacher and students) is altered due to the use of assistive computerbased systems. From the point of view of Figure 1, this mainly relates to the (multi-)mediality and (multi-)modality of communication processes. The chapter also bridges to the social complexity of communication as computer-assisted learning aims at autonomous learners who communicate not only more effectively with their teachers but 
also with each other. This is exemplified by peer-assisted learning, cooperative learning and learning in tandem that all are enhanced by technical means. The chapter provides an overview of the development of this field of research as well as a thorough exemplification in terms of computer-assisted language learning. The second part of the chapter is devoted to data driven learning and teaching thereby bridging to corpus-based approaches. It concludes with an overview of intelligent systems of CALL that integrate this area of application with intelligent systems as studied and built in computer science.

The topic of multimodal technical communication recurs in Chapter 18 ("Framing Multimodal Technical Communication"), in which Andy Lücking and Thies Pfeiffer concentrate on complexity issues in communication with speech and gesture, and the problem of integrating information from these two channels. Different forms of non-verbal behaviour are addressed in detail and the development of studies in this field is surveyed. The theoretical semiotic background is addressed in terms of sign types such as icon, index and symbol, and the interaction of modalities with media is discussed. A detailed overview of applications of technical communication is given, focussing specifically on devices for communicating gestural movement, such as eye-trackers and tracking gloves. Finally, issues of integration or fusion of multimodal data from different channels, as well as the fission or splitting of communication channels are discussed from technical and logical points of view.

Barrier-free communication is a field which has been seen as a small niche mainly for the sight and hearing impaired and those with serious illnesses and injuries, but in recent years the field has entered the mainstream of technical communication with human-machine interfaces. Petra Kubina and Andy Lücking discuss the state of the art in this area in Chapter 19 ("Barrier-free Communication"), pointing out areas such as the personal element of barriers to communication, with technical solutions for augmentative and alternative communication as well as social barriers to communication. Contexts for barrierfree communication such as the World-Wide Web are characterized in terms of perceivability, operability, understandability and robustness, and markup systems for structuring barrier-free communication data in the conventional web, the semantic web and the social web are discussed, including special issues like PDF readers. Finally a wide range 
of research directions, applications and tools are discussed, including examples such as the handheld Simputer developed in Bangalore for pre-literate communities, with icon input and speech synthesis output. Special techniques are also outlined. This includes tools for sonification and haptic input, through the treatment of sign languages to cochlear implants.

Recent developments in multimodal technical communication are treated by Stefan Kopp and Ipke Wachsmuth in Chapter 20 ("Artificial Interactivity"). Constraints on communication with embodied agents and avatars, also related to issues featured in Chapter 13 such as turn handling, timing and feedback are discussed in the context of interaction with a virtual human performing a construction task with instruction from a human interlocutor. The different levels of processing and integration of forms and meanings in multimodal behavior are discussed, and the specific issue of generating multimodal behavior is handled in detail, with special reference to the architecture of behavior production, from sensory data through feature extraction, segmentation and pattern recognition to interpretation of the behavior and its integration into existing knowledge representations.

Chapter 21 ("Ubiquitous Computing") by Codrina Lauth, Bettina Berendt, Bastian Pfleging \& Albrecht Schmidt extends the book's perspective on multimedial and multimodal technical communication. It deals with one of the most recent developments in this field of research that relates to the pervasiveness of computing in physically situated areas of human communication - that is, not in the virtual world of web-based communication (cf. Chapter 16) or, more specifically, social network sites (cf. Chapter 14). Instead, this chapter is about technical communication in situations of everyday communication in which visual, acoustic, haptic or even olfactoric channels are in use as transmission media. In principle, ubiquitous computing challenges the classical notion of human-computer interaction in that sensors can make the body as a whole an interface in technically enhanced everyday actions at any point in time at any location. This opens technical communication to new areas of application that have been widely closed for traditional approaches to text-based communication. With this development, we may enthusiastically state that we are at the beginning of a new area of technical communication (as explained and exemplified in this chapter) that covers the whole branch of 
semiosis, that is, of human sign formation based on whatever modality in whatever living conditions. In any event, any such assessment has to keep track with the current stage of development of these technologies. Chapter 21 does exactly this: it provides a definition of ubiquitous computing in conjunction with an overview of its current trends, exemplifications by means of short case studies as well as an overview of its underlying technologies and future challenges. In this way, it can be read as an entry point to one of the major developmental branches of technical communication.

The last chapter of Part III and also the last chapter of this handbook is Chapter 22 ("P2P-based Communication") by Gerhard Heyer, Florian Holz \& Sven Teresniak. It extends our series on future developments in technical communication. On the one hand, the chapter focuses on one of the traditional application areas of technical communication, that is, information retrieval (Baeza-Yates and Ribeiro-Neto, 1999; Melucci, Baeza-Yates, and Croft, 2011). However, it does so from the point of view of recent advancements in the theory of complex networks (Steyvers and Tenenbaum, 2005). The basic idea of this field of research is to profit from the peculiarities of social and semiotic networks in which neighbors tend to be linked according to similar or related interests, meanings or functions. Using this model, information retrieval in $\mathrm{P} 2 \mathrm{P}$ networks means giving up the notion of a centralized retrieval engine in order to rely instead on a network of semantically interlinked peers (software agents) whose networking is optimized to serve the retrieval needs. From this point of view, Chapter 22 mainly contributes to the social complexity of technically enhanced communication, however, it does so with a focus on monomodal written communication. Chapter 22 closes the handbook's thematic circle as it deals with an area of application that, at the beginning of this handbook, is described in terms of document authoring and representation.

\section{Acknowledgment}

We thank everyone who helped in realizing this handbook. First and foremost, this includes our authors who spent a lot of their academic time supporting this scientific endeavor. Our acknowledgement also includes the series editors as well as Barbara Karlson who accompanied 


\section{Introduction: Framing Technical Communication}

this project with great patience and understanding. 


\section{Notes}

1. See Lücking and Pfeiffer (2012) for a comprehensive consideration of these terms in the context of the notion of multimodal communication.

2. See Gibbon (2012) for a notion of modality that starts from a notion of communication as a pair of motor-sensory (output-input) organs, rather than separating production and reception.

3. Here one may also think of so called intelligent documents (Lobin, 1999).

4. See Menke (2012) for a similar approach using a classical model of signal transmission.

5. Ontologies concern the inference-driven processing of textual data and as such go beyond purely distributional approaches. The area of human-machine interaction based on artificial agents will profit especially from this research (Ferrucci, Brown, Chu-Carroll, Fan, Gondek, Kalyanpur, Lally, Murdock, Nyberg, Prager, Schlaefer, and Welty, 2010; Waltinger, Breuing, and Wachsmuth, 2011; Ovchinnikova, 2012).

6. Note that wikis currently evolve as a preferred information source of these approaches (Gabrilovich and Markovitch, 2009) so that the social complexity of wiki-based communication becomes an upcoming topic of text and web mining.

7. This is reflected, for example, by a recent Dagstuhl seminar on automation in digital preservation (Chanod, Dobreva, Rauber, and Ross, 2010). 



\section{Bibliography}

Ahn, Luis von (2008). "Human Computation." In: IEEE 24th International Conference on Data Engineering (ICDE 2008). IEEE, pp. 12 .

Altmann, Gabriel (1993). "Science and Linguistics." In: Contributions to Quantitative Linguistics. Ed. by Reinhard Köhler and Burghard B. Rieger. Dordrecht: Kluwer, pp. 3-10.

Altmann, Gabriel and Rüdiger Grotjahn (1988). "Linguistische Meßverfahren." In: Sociolinguistics. Soziolinguistik. An International Handbook of the Science of Language and Society. Ed. by U. Ammon, N. Dittmar, and K. J. Mattheier. Vol. 2. Berlin/New York: De Gruyter, pp. 1026-1039.

Baer, Daniel, Nicolai Erbs, Torsten Zesch, and Iryna Gurevych (2011). "Wikulu: An Extensible Architecture for Integrating Natural Language Processing Techniques with Wikis." In: Proceedings of the System Demonstrations of the 49th Annual Meeting of the Association for Computational Linguistics: Human Language Technologies.

Baeza-Yates, Ricardo and Berthier Ribeiro-Neto, eds. (1999). Modern Information Retrieval. Reading, Massachusetts: Addison-Wesley.

Berners-Lee, Tim, James Hendler, and Ora Lassila (2001). "The Semantic Web." In: Scientific American, pp. 34-43.

Brandes, Ulrik, Patrick Kenis, Jürgen Lerner, and Denise van Raaij (2009). "Network analysis of collaboration structure in Wikipedia." In: Proceedings of the 18th international conference on World wide web. WWW '09. Madrid, Spain: ACM, pp. 731-740.

Brinker, Klaus, Gerd Antos, Wolfgang Heinemann, and Sven F. Sager, eds. (2000). Text- und Gesprächslinguistik. Linguistics of Text and Conversation. Berlin/New York: De Gruyter.

Brinker, Klaus and Sven F. Sager (2006). Linguistische Gesprächsanalyse. Berlin: Erich Schmidt Verlag.

Brusilovsky, Peter (1996). "Methods and Techniques of Adaptive Hypermedia." In: User Modeling and User-Adapted Interaction 6.2-3, pp. $87-129$.

Bush, Vannevar (1945). "As We May Think." In: The Atlantic Monthly 176.1, pp. 101-108. 
Chanod, Jean-Pierre, Milena Dobreva, Andreas Rauber, and Seamus Ross, eds. (2010). Automation in Digital Preservation. Dagstuhl Seminar Proceedings 10291. Dagstuhl, Germany: Schloss Dagstuhl - Leibniz-Zentrum fuer Informatik, Germany.

Chiarcos, Christian, Sebastian Nordhoff, and Sebastian Hellmann, eds. (2012). Linked Data in Linguistics. Berlin: Springer, pp. 15-23.

Dang, Jing, Anthony Brabazon, David Edelman, and Michael O'Neill (2009). "An Introduction to Natural Computing in Finance." In: Applications of Evolutionary Computing. Ed. by M. Giacobini, A. Brabazon, S. Cagnoni, G. A. Di Caro, R. Drechsler, A. Ekart, A. I. Esparcia-Alcazar, M. Farooq, A. Fink, J. McCormack, M. O'Neill, J. Romero, F. Rothlauf, G. Squillero, S. Uyar, and S. Yang. Vol. 5484. Lecture Notes in Computer Science. Springer, pp. 182-192.

Diewald, Nils (2012). "Decentralized social network sites." In: Handbook of Technical Communication. Ed. by Alexander Mehler, Laurent Romary, and Dafydd Gibbon. Berlin/Boston: De Gruyter, pp.

Dürscheid, Christa (2003). "Medienkommunikation im Kontinuum von Mündlichkeit und Schriftlichkeit. Theoretische und empirische Probleme." In: Zeitschrift für angewandte Linguistik 38, pp. 37-56.

Eco, Umberto (1988). Über Spiegel und andere Phänomene. München: Hanser.

Egozi, Ofer, Shaul Markovitch, and Evgeniy Gabrilovich (2011). "ConceptBased Information Retrieval using Explicit Semantic Analysis." In: ACM Transactions on Information Systems 29.2.

Engelbart, Douglas C. (1995). "Toward augmenting the human intellect and boosting our collective IQ." In: Communications of the ACM 38 (8), pp. $30-32$.

Feldman, Ronen and James Sanger (2007). The Text Mining Handbook. Advanced Approaches in Analyzing Unstructured Data. Cambridge: Cambridge University Press.

Ferrucci, David, Eric Brown, Jennifer Chu-Carroll, James Fan, David Gondek, Aditya A. Kalyanpur, Adam Lally, J. William Murdock, Eric Nyberg, John Prager, Nico Schlaefer, and Chris Welty (2010). "Building Watson: An Overview of the DeepQA Project." In: AI Magazine 31.3, pp. 59-79.

Gabrilovich, Evgeniy and Shaul Markovitch (2009). "Wikipedia-based Semantic Interpretation for Natural Language Processing." In: Journal of Artificial Intelligence Research, pp. 443-498. 
Gibbon, Dafydd (2012). "Resources for technical communication systems." In: Handbook of Technical Communication. Ed. by Alexander Mehler, Laurent Romary, and Dafydd Gibbon. Berlin/Boston: De Gruyter, pp.

Gibbon, Dafydd, Inge Mertins, and Roger K. Moore, eds. (2000). Handbook of Multimodal and Spoken Dialogue Systems. Boston/Dordrecht/London: Kluwer.

Ginzburg, Jonathan and Raquel Fernandez (2005). "Action at a distance: the difference between dialogue and multilogue." In: Proceedings of the 9th Workshop on the Semantics and Pragmatics of Dialogue (Dialor'05), Nancy, France.

Gómez, Vicenç, Hilbert J. Kappen, and Andreas Kaltenbrunner (2011). "Modeling the structure and evolution of discussion cascades." In: Proceedings of the 22nd ACM conference on Hypertext and hypermedia. HT '11. Eindhoven, The Netherlands: ACM, pp. 181-190.

Hammwöhner, Rainer (2007). Semantic Wikipedia - Checking the Premises. http://epub.uni-regensburg.de/15593/.

Harris, Zellig S. (1954). "Distributional Structure.” In: Word 10, pp. 146162.

Hess-Lüttich, Ernst W. B. and Dagmar Schmauks (2004). "Multimediale Kommunikation." In: Semiotik: ein Handbuch zu den zeichentheoretischen Grundlagen von Natur und Kultur. Ed. by Roland Posner, Klaus Robering, and Thomas A. Seboek. Vol. 4. Handbücher zur Sprach- und Kommunikationswissenschaft. Berlin: de Gruyter. Chap. XV. Ausgewählte Gegenstände der Semiotik / Selected Topics of Semiotics, pp. 3487-3503.

Kendon, Adam (2004). Gesture: Visible Action as Utterance. Cambridge, MA: Cambridge University Press.

Koch, Peter and Wulf Oesterreicher (2001). "Langage écrit et langage parlé." In: Lexikon der romanistischen Linguistik. Ed. by Günter Holtus, Michael Metzeltin, and Christian Schmitt. Vol. I/1. Tübingen, Germany: Niemeyer, pp. 584-627.

Kubina, Petra and Andy Lücking (2012). "Barrier-free communication." In: Handbook of Technical Communication. Ed. by Alexander Mehler, Laurent Romary, and Dafydd Gibbon. Berlin/Boston: De Gruyter, pp.

Ladkin, Peter (2012). "Verbal communication protocols in safety-critical system operations." In: Handbook of Technical Communication. Ed. 
by Alexander Mehler, Laurent Romary, and Dafydd Gibbon. Berlin/Boston: De Gruyter, pp.

Landauer, Thomas K. and Susan T. Dumais (1997). "A Solution to Plato's Problem: The Latent Semantic Analysis Theory of Acquisition, Induction, and Representation of Knowledge." In: Psychological Review 104.2, pp. 211-240.

Laniado, D., R. Tasso, Y. Volkovich, and A. Kaltenbrunner (2011). "When the Wikipedians Talk: Network and Tree Structure of Wikipedia Discussion Pages." In: Proceedings of the 5th International AAAI Conference on Weblogs and Social Media.

Lauth, Codrina, Bettina Berendt, Albrecht Schmidt, and Bastian Pfleging (2012). "Ubiquitous computing." In: Handbook of Technical Communication. Ed. by Alexander Mehler, Laurent Romary, and Dafydd Gibbon. Berlin/Boston: De Gruyter, pp.

Lee, Christopher A. (2012). "Digital curation as communication." In: Handbook of Technical Communication. Ed. by Alexander Mehler, Laurent Romary, and Dafydd Gibbon. Berlin/Boston: De Gruyter, pp.

Leuf, Bo and Ward Cunningham (2001). The Wiki Way. Quick Collaboration on the Web. Boston: Addison Wesley.

Lobin, Henning (1999). "Intelligente Dokumente. Linguistische Repräsentation komplexer Inhalte für die hypermediale Wissensvermittlung." In: Text im digitalen Medium. Linguistische Aspekte von Textdesign, Texttechnologie und Hypertext Engineering. Ed. by Henning Lobin. Opladen: Westdeutscher Verlag, pp. 89-119.

Lücking, Andy, Alexander Mehler, and Peter Menke (2008). "Taking Fingerprints of Speech-and-Gesture Ensembles: Approaching Empirical Evidence of Intrapersonal Alignment in Multimodal Communication." In: LONDIAL 2008: Proceedings of the 12th Workshop on the Semantics and Pragmatics of Dialogue (SEMDIAL). King's College London, pp. 157-164.

Lücking, Andy and Thies Pfeiffer (2012). "Framing multi-modal technical communication. With Focal Points in Speech-Gesture-Integration and Gaze Recognition." In: Handbook of Technical Communication. Ed. by Alexander Mehler, Laurent Romary, and Dafydd Gibbon. Berlin/Boston: De Gruyter, pp.

Mehler, Alexander (2009). "Artifizielle Interaktivität. Eine semiotische Betrachtung." In: Medienwandel als Wandel von Interaktionsformen 
- von frühen Medienkulturen zum Web 2.0. Ed. by Tilmann Sutter and Alexander Mehler. Wiesbaden: VS.

Mehler, Alexander and Tilmann Sutter (2008). "Interaktive Textproduktion in Wiki-basierten Kommunikationssystemen." In: Kommunikation, Partizipation und Wirkungen im Social Web - Weblogs, Wikis, Podcasts und Communities aus interdisziplinärer Sicht. Ed. by Ansgar Zerfaß, Martin Welker, and Jan Schmidt. Köln: Herbert von Halem, pp. 267-300.

Melucci, Massimo, Ricardo Baeza-Yates, and W. Bruce Croft, eds. (2011). Advanced Topics in Information Retrieval. Vol. 33. The Information Retrieval Series. Berlin: Springer.

Menke, Peter (2012). "Evaluation of technical communication." In: Handbook of Technical Communication. Ed. by Alexander Mehler, Laurent Romary, and Dafydd Gibbon. Berlin/Boston: De Gruyter, pp.

Nelson, Theodor Holm (1995). "The heart of connection: hypermedia unified by transclusion." In: Communications of the ACM 38 (8), pp. 31-33.

Newman, Mark E. J. (2010). Networks: An Introduction. Oxford: Oxford University Press.

O'Donnell, Michael, Chris Mellish, Jon Oberlander, and Alisair Knott (2001). "ILEX: An Architecture for a Dynamic Hypertext Generation System." In: Natural Language Engineering 7, pp. 225-250.

Ovchinnikova, Ekaterina (2012). Integration of World Knowledge for Natural Language Understanding. Paris: Atlantis Press.

Paaß, Gerhard (2012). "Document classification, information retrieval, text and web mining." In: Handbook of Technical Communication. Ed. by Alexander Mehler, Laurent Romary, and Dafydd Gibbon. Berlin/Boston: De Gruyter, pp.

Pickering, Martin J. and Simon Garrod (2004). "Toward a mechanistic psychology of dialogue." In: Behavioral and Brain Sciences 27, pp. 169-226.

Posner, Roland, Klaus Robering, and Thomas A. Seboek, eds. (1996). Semiotik: ein Handbuch zu den zeichentheoretischen Grundlagen von Natur und Kultur. Vol. 1. Handbücher zur Sprach- und Kommunikationswissenschaft. Berlin: de Gruyter.

Rickheit, Gert (2005). "Alignment und Aushandlung im Dialog." In: Zeitschrift für Psychologie 213.3, pp. 159-166. 
Rieger, Burghard B. (2003). "Semiotic Cognitive Information Processing: Learning to Understand Discourse. A Systemic Model of Meaning Constitution." In: Adaptivity and Learning. An Interdisciplinary Debate. Ed. by R. Kühn, R. Menzel, W. Menzel, U. Ratsch, M. M. Richter, and I. O. Stamatescu. Berlin: Springer, pp. 347-403.

Ruiter, Jan Peter de, Stéphane Rossignol, Louis Vuurpijl, Douglas Cunningham, and Willem J. M. Levelt (2003). "SLOT: A research platform for investigating multimodal communication." In: Behavior Research Methods 35 (3), pp. 408-419.

Sager, Sven F. (1997). "Intertextualität und die Interaktivität von Hypertexten." In: Textbeziehungen: linguistische und literaturwissenschaftliche Beiträge zur Intertextualität. Ed. by Josef Klein and Ulla Fix. Tübingen: Stauffenburg, pp. 109-123.

Sasaki, Felix and Arle Lommel (2012). "Multilingual computing." In: Handbook of Technical Communication. Ed. by Alexander Mehler, Laurent Romary, and Dafydd Gibbon. Berlin/Boston: De Gruyter, pp.

Schaffert, Sebastian, Diana Bischof, Tobias Bürger, Andreas Gruber, Wolf Hilzensauer, and Sandra Schaffert (2006). "Learning with Semantic Wikis." In: Proceedings of SemWiki2006 Workshop "From Wiki to Semantics", pp. 109-123.

Steels, Luc and Peter Hanappe (2006). "Interoperability through Emergent Semantics. A Semiotic Dynamics Approach." In: Journal on Data Semantics VI, pp. 143-167.

Stegbauer, Christian (2009). Wikipedia: Das Rätsel der Kooperation. Wiesbaden: VS.

Steyvers, Mark and Josh Tenenbaum (2005). "The large-scale structure of semantic networks: Statistical analyses and a model of semantic growth." In: Cognitive Science 29.1, pp. 41-78.

Storrer, Angelika (2002). "Coherence in Text and Hypertext." In: Document Design 3.2, pp. 156-168.

Turney, Peter D. and Patrick Pantel (2010). "From Frequency to Meaning: Vector Space Models of Semantics." In: Journal of Artificial Intelligence Research 37, pp. 141-188.

Völkel, M., M. Krötzsch, D. Vrandecic, H. Haller, and R. Studer (2006). "Semantic Wikipedia." In: Proceedings of the 15th International Conference on World Wide Web (WWW'06), Edinburgh, Scotland, May 23 - 26. New York: ACM Press, pp. 585-594. 
Waltinger, Ulli and Alexa Breuing (2012). "Internet-based communication." In: Handbook of Technical Communication. Ed. by Alexander Mehler, Laurent Romary, and Dafydd Gibbon. Berlin/Boston: de Gruyter, pp.

Waltinger, Ulli, Alexa Breuing, and Ipke Wachsmuth (2011). "Interfacing Virtual Agents With Collaborative Knowledge: Open Domain Question Answering Using Wikipedia-based Topic Models." In: Proceedings of the International Joint Conference on Artificial Intelligence (IJCAI 2011), July, 16-22, 2011, Barcelona, Spain.

Wasserman, Stanley and Katherine Faust (1999). Social Network Analysis. Methods and Applications. Cambridge: Cambridge University Press.

Wattenberg, Martin, Fernanda B. Viégas, and Katherine J. Hollenbach (2007). "Visualizing Activity on Wikipedia with Chromograms." In: Human-Computer Interaction - INTERACT 2007, 11th IFIP TC 13 International Conference, Rio de Janeiro, Brazil, September 10-14, 200\%, Proceedings, Part II. Ed. by Maria Cecília Calani Baranauskas, Philippe A. Palanque, Julio Abascal, and Simone Diniz Junqueira Barbosa. Vol. 4663. LNCS. Springer, pp. 272-287.

Wehner, Josef (1997). "Interaktive Medien - Ende der Massenkommunikation." In: Zeitschrift für Soziologie 26.4, pp. 96-11.

Weidenmann, Bernd (1997). "Multicodierung und Multimodalität im Lernprozess." In: Information und Lernen mit Multimedia. Ed. by Ludwig J. Issing and Paul Klimsa. Weinheim: Beltz, pp. 45-62.

Wilks, Yorick (1997). "Information Extraction as a Core Language Technology." In: Information Extraction: a Multidisciplinary Approach to an Emerging Information Technology. Proceedings of the International Summer School (SCIE-97), Frascati, Italy. Ed. by Maria Terese Pazienza. Lecture notes in computer science 1299. Berlin: Springer, pp. 1-9.

Zerubavel, Eviatar (2003). Time Maps: Collective Memory and the Social Shape of the Past. Berkley: University of California Press. 